\title{
Knowledge of dietary and behaviour-related determinants of non-communicable disease in urban Senegalese women
}

\author{
Michelle Holdsworth* , Francis Delpeuch, Edwige Landais, Agnès Gartner, \\ Sabrina Eymard-Duvernay and Bernard Maire \\ UR 106 Nutrition, Alimentation et Sociétés, WHO Collaborating Centre in Human Nutrition, \\ Institut de Recherche pour le Développement (IRD), BP 64501, F-34394 Montpellier Cedex 5, France
}

Submitted 31 August 2005: Accepted 31 January 2006

\begin{abstract}
Objective: To assess knowledge of dietary and behaviour-related determinants of non-communicable disease (NCD) of urban Senegalese women.

Design: A cross-sectional, population study using an interviewer-administered knowledge questionnaire, developed and validated for this study. The questionnaire consisted of 24 items with six scores measuring knowledge of: (1) diet- and behaviour-related causes of NCD; (2) diet quality-NCD relationship; (3) fruit and vegetable link with NCD; (4) health consequences of obesity; (5) causes of cardiovascular disease (CVD); and (6) causes of certain cancers.

Subjects: A random sample of 301 women aged 20-50 years.

Results: The knowledge scores developed suggest that the health consequences of obesity (mean score of 65.4\%) were best understood followed by causes of CVD (mean score of 60.6\%), because obesity, smoking, high blood cholesterol and dietary fat were well recognised as risk factors for CVD. Subjects scored least for their knowledge of the protective effect of fruit and vegetables (mean score of 19.9\%). Knowledge of causes of certain cancers (mean score of 36.1\%) was also low. Women who worked outside the home had better knowledge for two scores but otherwise no relationship was found between knowledge and literacy, formal education or body mass index.

Conclusions: Findings suggest reasonable overall knowledge concerning diet and behaviour with NCD, especially given the relatively new context of the obesity epidemic in Senegal. However, there was poor knowledge of the benefit of eating fruit and vegetables and other preventable causes of certain cancers. Education targeting the benefits of vegetables and fruit may have the greatest impact on NCD prevention.
\end{abstract}

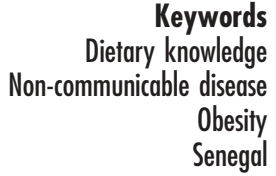

Non-communicable diseases (NCDs) including cardiovascular disease (CVD), type 2 diabetes, cancers and obesityrelated conditions now account for some $60 \%$ of global deaths and almost half (47\%) of the global burden of disease $^{1}$. NCDs no longer principally touch high-income countries: low- and medium-income countries are increasingly implicated ${ }^{2,3}$, as highlighted in the World Health Organization's (WHO) Global Strategy on Diet, Physical Activity and Health ${ }^{1}$. The WHO strategy addresses diet and physical activity, two of the major risk factors responsible for the growing burden of NCDs, and emphasises the need to limit consumption of salt, saturated fats and refined sugars, and to increase consumption of vegetables and fruit and levels of physical activity. It also identifies the need to address the role of consumer education and communication.

One of the obstacles to slowing down the increase of obesity and nutrition-related chronic disease in some low- and medium-income countries could be a lack of individuals' knowledge of their causes and relationship to factors such as poor diet and lack of physical activity. Certainly, lack of awareness of healthy behaviours by the general public in Africa is perceived as one contributory factor to $\mathrm{NCDs}^{4}$ and it has been proposed that the low level of formal education, especially in women, justifies emphasis on knowledge dissemination in Africa ${ }^{5}$. Even so, there are few studies in sub-Saharan Africa to date to substantiate this, which is to be expected given that NCDs are a relatively recent public health problem, with the exception of South Africa ${ }^{6}$.

Although some dietary knowledge is necessary to persuade individuals to choose health-promoting behaviours $^{7-9}$, it is acknowledged that it still remains only one predisposing factor influencing the complex behaviour of eating $^{10}$, alongside factors such as attitudes, risk perception and social norms ${ }^{11}$. Accurate nutritional knowledge 
may be particularly important when individuals are ready to make dietary changes ${ }^{7}$ and when combined with behavioural and motivational strategies ${ }^{12}$. Early studies have been sceptical about the potential changes in behaviour that could result from enhancing knowledge, but the tools used to assess dietary knowledge have often been imprecise and rarely tested for their validity and reliability in measuring what was intended, making it difficult to judge if the findings were accurate. Nutrition knowledge tools have since been developed ${ }^{13-15}$ which appear to be more sensitive at measuring dietary knowledge, as well as knowledge of the diet-NCD relationship. However, these do not contain the level of precision on the dietary and behaviour-NCD relationship needed for the current study.

Almost half of Senegal's inhabitants are urban, a figure which is set to increase, and a quarter of the Senegalese population lives in the region of the capital, Dakar. Obesity prevalence of women in a district of Dakar has doubled since 1996 and has recently been estimated at $18.6 \%{ }^{16}$. The likelihood that obesity is set to stay means that the wide range of determinants of obesity in this context need to be explored, including understanding of the causes and consequences of NCD, to help inform future preventive strategies. The purpose of the current study of these urban Senegalese women was to assess their knowledge of dietary and behaviour-related determinants of NCD, to identify gaps in knowledge that could be the target for future public health nutrition programmes.

\section{Methods}

\section{Target population and sampling}

The target population was Senegalese women aged 20-50 years living in Dakar. The sampling frame was the district of Pikine Ancien. This district (population of about 100000 inhabitants) was chosen because it is the most diverse in terms of coverage of all sociodemographic groups in the large town of Pikine (population of about 1 million inhabitants). Although we cannot refer to how representative our sample is in statistical terms on a wider scale, we can say that the district of Pikine Ancien is by no means atypical of the rest of Pikine or Dakar in sociodemographic or cultural terms.

Approval was granted in April 2003 by the local ethical committee (Chief Medical Officer, Pikine District). Informed oral consent was obtained from each subject during recruitment. Fieldwork was carried out during April-June 2003 in Wolof (language understood by all). All interviews were conducted verbally by the same trained female interviewer, supervised by a female researcher at all times (E.L.).

Using a recent map, 310 compounds (a dwelling that several households may share) were randomly selected using random number tables; the additional 10 compounds were sampled to provide a replacement pool in case of refusals or where compounds no longer existed. One household (if the compound comprised more than one) was randomly selected using a die at the interior of the compound. Only one woman was randomly selected from this household to avoid any bias from potential clustering of knowledge within the same compound. On average 10 people comprise a household in Pikine.

\section{Development and validation of the questionnaire}

The questionnaire was composed of 24 items in total (Table 1). All items were developed specifically for this study, except for items 16 and 17 which were based on those used previously ${ }^{17}$. Questions were chosen on areas of knowledge with high scientific consensus about the causality of NCD, so that items were clearly unequivocally true or false. Areas of knowledge of modifiable behaviours were targeted to be able to inform intervention programmes. The questionnaire was pre-tested for face validity with 10 Senegalese women drawn from a population within Dakar. It was developed in French and was translated into Wolof, which was the language used for interviews, and then translated back into French to check that none of the meaning had been lost. Piloting ensured that ambiguous and confusing questions were identified and re-phrased. Although questions where the correct response was 'false' were included the piloting stage, only one such question was retained after piloting. This was for several reasons. First, in terms of language, as negatively phrased questions were not well understood and some of these were perceived as patronising by the women as too obviously incorrect. Second, it has been recognised that adults with lower cognitive skills/formal educational level are more easily confused by questions where they have to answer 'no' for a correct response, leading to lower validity coefficients ${ }^{18}$, and simply reversing the polarity of an item does not necessarily reverse its meaning.

The items were used to construct six knowledge domain scores: Score 1 - dietary and behaviour-related causes of NCD; Score 2 - diet quality-NCD relationship; Score 3 fruit and vegetables link with NCD; Score 4 - health consequences of obesity; Score 5 - causes of CVD; and Score 6 - causes of certain cancers; all items were used for more than one of these knowledge domains (Table 2). These scores are mutually exclusive in their ability to measure their domain of knowledge. The six knowledge domain scores passed the criteria for internal consistency, item discrimination and item difficulty in the final sample of 301 women (Table 2).

- Internal consistency: The reliability of each set of items in measuring each domain indicated that item-to-item correlation was acceptable (Table 2) using Cronbach's $\alpha$ for all domain scores except Score $6(\alpha=0.69)$, which was marginally below the suggested cut-off point of 0.7 . 
Table 1 Questionnaire items

\begin{tabular}{l}
\hline No \\
1. Eating a lot of fat can contribute to heart problems \\
2. Eating a lot of fat can contribute to obesity \\
3. Eating a lot of fat can contribute to certain cancers \\
4. Eating a lot of sugar can contribute to heart problems \\
5. Eating a lot of sugar can contribute to obesity \\
6. Eating a lot of salt can contribute to heart problems \\
7. Eating a lot of salt can contribute to certain cancers \\
8. Low intake of fruit can contribute to heart problems \\
9. Low intake of fruit can contribute to obesity \\
10. Low intake of fruit can contribute to certain cancers \\
11. Low intake of vegetables can contribute to heart problems \\
12. Low intake of vegetables can contribute to obesity \\
13. Low intake of vegetables can contribute to certain cancers \\
14. Eating too much food can contribute to obesity \\
15. Obesity increases the risk of developing diabetes \\
16. Obesity increases risk of breast cancer after the menopause \\
17. Obesity increases the risk of developing bowel cancer \\
18. Obesity increases risk of developing hypertension \\
19. Weight increase gradually increases risk of heart problems \\
20. Lack of physical activity can contribute to obesity \\
21. Lack of physical activity can contribute to heart problems \\
22. Obesity can contribute to heart problems \\
23. High blood cholesterol can contribute to heart problems \\
24. Smoking can contribute to heart disease
\end{tabular}

All responses are true except item 4.

Items presented here in the order that they appeared on the questionnaire.

Even so, this was retained because item discrimination and difficulty, as well as content validity, were convincing.

- Item discrimination: The ability of each individual item to discriminate between women with different levels of knowledge, i.e. highest $27 \%$ versus lowest $27 \%$, was measured by correlating the response on each item with the score ${ }^{19}$. All items had an item-to-item score correlation of $>0.2$, below which items are considered too weak ${ }^{18}$.

- Item difficulty: Eighteen of the 24 items (Table 2) fell within the recommended range of $20-80 \%$ of correct responses ${ }^{14,20}$. Three of the remaining six items were too easy (88.4\% correctly answered 'obesity increases risk of hypertension', $81.1 \%$ that 'lack of physical activity can contribute to obesity' and $81.4 \%$ that 'eating a lot of fat can contribute to heart problems') and three were too difficult (13.3\% correctly answered 'low intake of fruit can contribute to certain cancers', $17.9 \%$ correctly answered 'low intake of vegetables can contribute to obesity' and 14.6\% correctly answered 'low intake of vegetables can contribute to certain cancers'). Even so, these items were retained on the grounds of content validity, as they were testing a crucial facet of the dietNCD relationship not otherwise addressed in the questionnaire.

\section{Demograpbic, socio-economic and nutritional measures}

Age, written literacy, education level, employment status and ethnicity were assessed. Economic level was estimated by the presence in the compound of dwelling utilities (electricity, running water), appliances (refrigerator, freezer) and ability to purchase staple food in advance. A synthetic index of economic level was constructed with multiple correspondence analyses on

Table 2 Internal reliability of knowledge domain scores

\begin{tabular}{|c|c|c|c|c|}
\hline Knowledge domain score & Items* & No. of items & Cronbach's $\alpha$ & $\begin{array}{l}\text { Item discrimination } \\
\text { range }\end{array}$ \\
\hline Score 1: Diet- and behaviour-related causes of NCD & $1-14,20,21,23,24$ & 18 & 0.84 & $0.20-0.79$ \\
\hline Score 2: Diet quality-NCD relationship & $1-13$ & 13 & 0.84 & $0.32-0.69$ \\
\hline Score 3: Fruit and vegetable-NCD relationship & $8-13$ & 6 & 0.83 & $0.50-0.79$ \\
\hline Score 4: Health consequences of obesity & $15-19,22$ & 6 & 0.72 & $0.28-0.73$ \\
\hline Score 5: Causes of CVD & $1,4,6,8,11,18,19,21-24$ & 11 & 0.79 & $0.36-0.85$ \\
\hline Score 6: Causes of certain cancers & $3,7,10,13,16,17$ & 6 & 0.69 & $0.32-0.84$ \\
\hline
\end{tabular}

NCD - non-communicable disease; CVD - cardiovascular disease.

* Item numbers correspond to those listed in Table 1. 
this matrix of indicator variables, based on an approach developed previously ${ }^{21}$. The first principal component of the correspondence analysis displayed a gradient of household wealth. The economic index developed classified households into three distinct groups of 'low', 'average' and 'high' economic level. Body mass index (BMI) was assessed from measured weight and height; weight was measured using scales accurate to $100 \mathrm{~g}$, which were verified daily, and height was measured using a portable height measurer to the nearest $\mathrm{mm}$. BMI data were classified into two groups for cross-tabulations: 'underweight/normal' and 'overweight/obese'.

\section{Data management and analyses}

Data from questionnaires were entered twice by the same investigator (E.L.) in two separate files using EpiData (version 3; The EpiData Association, Odense, Denmark), their output was then compared by another researcher (A.G.) and the corrections identified and inputted. SPSS (version 11.5; SPSS Inc., Chicago, IL, USA) was used for descriptive analyses and ADE- $4^{22}$ was used for correspondence analysis when developing the economic index. Knowledge domain scores were attributed as follows: correct response $=1$; incorrect response $=0$; unsure/don't know $=0$. The 'unsure/don't know' category was included to discourage bias from guessing ${ }^{13}$. Knowledge scores were standardised as percentages so that they could be compared. In the development of the scores, obesity was treated as an NCD, not one of the determinants. The relationship between the six knowledge scores and sociodemographic variables (economic level, employment status, age, BMI, literacy, ethnicity and education level) were investigated using the Kruskal-Wallis test. Differences were considered statistically different at the $P<0.05$ level.

\section{Results}

\section{Characteristics of the sample}

The overall response rate was $97.1 \%$, as 301 of the 310 randomly selected women agreed to participate and form the final sample. Wolof was the major ethnic group (45.2\%) (Table 3). About two-thirds of women had received either no school education (35.9\%) or only primary school level education (32.9\%), almost over half of respondents reported being illiterate (47.8\%). Only a quarter of women reported working outside the home (26.1\%), and almost half of these (46.3\%) were market/shop keepers. The majority of households had electricity (91.0\%) and running water (89.7\%) and nearly half owned a refrigerator (48.8\%). Almost half the women (45.2\%) were classified as either overweight (26.6\%) or obese (18.6\%).

\section{Knowledge of diet and behavioural determinants of NCDs}

Knowledge was understood better for the dietary fatNCD relationship (Table 4) as the percentage of correct
Table 3 Characteristics of the sample $(n=301$ women)

\begin{tabular}{|c|c|}
\hline Characteristic & $\%$ \\
\hline \multicolumn{2}{|l|}{ Age (years) } \\
\hline $20-29$ & 40.9 \\
\hline $30-39$ & 36.5 \\
\hline $40-49$ & 22.6 \\
\hline \multicolumn{2}{|l|}{ Education level } \\
\hline None/partial primary & 48.9 \\
\hline Full primary & 32.9 \\
\hline At least secondary & 18.2 \\
\hline Written literacy & 52.2 \\
\hline \multicolumn{2}{|l|}{ Employment } \\
\hline Unemployed & 73.9 \\
\hline Shop/market keeper & 12.4 \\
\hline Dressmaker & 4.7 \\
\hline Other & 9.0 \\
\hline \multicolumn{2}{|l|}{ Ethnicity } \\
\hline Wolof & 45.2 \\
\hline Toucouleur/Peuhl & 28.5 \\
\hline Other & 26.2 \\
\hline \multicolumn{2}{|l|}{ Economic level } \\
\hline Electricity & 91.0 \\
\hline Water & 89.7 \\
\hline Refrigerator & 48.8 \\
\hline Freezer & 13.6 \\
\hline Purchase food staple in advance* & 57.3 \\
\hline \multicolumn{2}{|l|}{ Economic index } \\
\hline Low & 34.9 \\
\hline Average & 28.3 \\
\hline High & 36.8 \\
\hline \multicolumn{2}{|l|}{ Body mass index $\left(\mathrm{kg} \mathrm{m}^{-2}\right)$} \\
\hline$<18.5$ (underweight) & 5.3 \\
\hline $18.5-24.9$ (normal) & 49.5 \\
\hline 25.0-29.9 (overweight) & 26.6 \\
\hline$\geq 30.0$ (obese) & 18.6 \\
\hline
\end{tabular}

${ }^{*}$ At the beginning of the month.

responses ranged between 49.8 and $81.4 \%$ according to the item, whereas they were lower for the sugar-NCD link (correct responses ranged from 32.6 to 54.5\%). For example, the item 'eating a lot of fat can contribute to heart problems' was widely understood, with $81.4 \%$ of women answering correctly. Knowledge of the saltNCD relationship was slightly lower, with correct responses in the range of 26.3-56.3\%. However, the least well understood association was that between NCDs and low intake of fruit and vegetables (correct responses ranging between 13.3 and 31.6\%); for example, only $13.3 \%$ of women correctly answered that a 'low intake of fruit can contribute to certain cancers'.

Almost three-quarters of women were aware that eating too much food could cause obesity, whereas less were aware of the contribution of eating a lot of sugar (54.5\%) and fat (59.5\%) to obesity development (Table 4). Obesity as a risk factor for CVD was well understood, with $88.4 \%$ of women acknowledging that obesity could lead to hypertension and $75.4 \%$ agreeing that 'weight increase gradually throughout life increases risk of developing heart problems'. Smoking as a CVD risk factor was also well understood ( $73.8 \%$ correct), as was fat consumption 
Table 4 Correct responses for individual knowledge items ( $n=301$ women)

\begin{tabular}{|c|c|c|c|}
\hline Item & $\begin{array}{c}\% \\
\text { correct }\end{array}$ & $\begin{array}{c}\% \\
\text { incorrect }\end{array}$ & $\begin{array}{c}\% \\
\text { unsure }\end{array}$ \\
\hline 1. Eating a lot of fat can contribute to heart problems & 81.4 & 5.6 & 13.0 \\
\hline 2. Eating a lot of fat can contribute to obesity & 59.5 & 20.6 & 19.9 \\
\hline 3. Eating a lot of fat can contribute to certain cancers & 49.8 & 11.0 & 39.2 \\
\hline 4. Eating a lot of sugar can contribute to heart problems & 32.6 & 32.6 & 24.3 \\
\hline 5. Eating a lot of sugar can contribute to obesity & 54.5 & 26.9 & 18.6 \\
\hline 6. Eating a lot of salt can contribute to heart problems & 56.3 & 24.7 & 19.0 \\
\hline 7. Eating a lot of salt can contribute to certain cancers & 26.3 & 34.3 & 39.3 \\
\hline 8. Low intake of fruit can contribute to heart problems & 31.6 & 40.2 & 28.2 \\
\hline 9. Low intake of fruit can contribute to obesity & 20.6 & 61.1 & 18.3 \\
\hline 10. Low intake of fruit can contribute to certain cancers & 13.3 & 53.8 & 32.9 \\
\hline 11. Low intake of vegetables can contribute to heart problems & 22.6 & 50.2 & 27.2 \\
\hline 12. Low intake of vegetables can contribute to obesity & 17.9 & 64.8 & 17.3 \\
\hline 13. Low intake of vegetables can contribute to certain cancers & 14.6 & 54.8 & 30.6 \\
\hline 14. Eating too much food can contribute to obesity & 71.1 & 26.9 & 2.0 \\
\hline 15. Obesity increases the risk of developing diabetes & 51.2 & 41.2 & 7.6 \\
\hline 16. Obesity increases risk of breast cancer after the menopause & 52.8 & 10.3 & 36.9 \\
\hline 17. Obesity increases the risk of developing bowel cancer & 59.1 & 10.6 & 30.2 \\
\hline 18. Obesity increases risk of developing hypertension & 88.4 & 5.6 & 6.0 \\
\hline 19. Weight increase gradually increases risk of heart problems & 75.4 & 7.0 & 17.6 \\
\hline 20. Lack of physical activity can contribute to obesity & 81.1 & 15.6 & 3.3 \\
\hline 21. Lack of physical activity can contribute to heart problems & 58.8 & 23.3 & 17.9 \\
\hline 22. Obesity can contribute to heart problems & 65.4 & 15.3 & 19.3 \\
\hline 23. High blood cholesterol can contribute to heart problems & 69.8 & 8.6 & 21.6 \\
\hline 24. Smoking can contribute to heart disease & 73.8 & 13.0 & 13.3 \\
\hline
\end{tabular}

Items presented here in the order that they appeared on the questionnaire.

(81.4\% correct). However, eating a lot of fat was seen to have more impact on CVD risk than development of obesity $(59.5 \%$ correct). Over two-thirds of women (Table 4) were aware that high blood cholesterol was a CVD risk factor. A lack of physical activity was seen as having more impact in contributing to obesity (81.1\% correct) than CVD development (58.8\% correct).

\section{Knowledge of the bealth consequences of obesity}

The effect of obesity on CVD was generally well understood (Table 4), but its impact on certain cancers was less well known, less so for breast cancer $(52.8 \%$ correct) than for bowel cancer $(59.1 \%$ correct). Over half of the sample (59.1\%) was aware that obesity could impact on life expectancy. However, the least well understood health consequence of obesity was its potential impact on the development of diabetes (51.2\% correct).

\section{Knowledge scores}

The knowledge scores developed (Table 5) suggest that the health consequences of obesity (mean score of 65.4\%) were best understood. Amongst the determinants of NCDs, the causes of certain cancers were less well understood than for CVD (Table 5). Knowledge of the protective effect of vegetables and fruit was least well known (mean score of 19.9\%). No relationship was found between knowledge scores and women's age, economic level, literacy, education level, BMI or ethnicity. However, women who worked outside the home had significantly better knowledge than those unemployed for Scores 1 and $2(P<0.05)$.

\section{Discussion}

The purpose of this study was to assess knowledge of dietary and behaviour-related determinants of NCDs in urban Senegalese women, to identify gaps in knowledge that could be the target for future public health nutrition programmes. Overall, the findings suggest reasonable overall knowledge concerning dietary and behaviour-related causes of NCDs, especially given the relatively new context of the obesity epidemic in Senegal. However, there was particularly poor knowledge of the benefit of eating plenty of vegetables and fruit in preventing obesity, CVD and certain cancers. Knowledge of the potential for preventing cancers was least understood, whereas it was widely understood for CVD. These trends are similar to those reported in previous studies conducted in high-income countries ${ }^{8,23}$.

The knowledge scores constructed suggest that women had less knowledge about the causes of NCDs than about the consequences of obesity. This could result from their direct or indirect life experiences of the consequences of obesity. However, the non-dietary causes of NCDs appear to be better understood than the dietary risk factors alone, which suggests that education should particularly focus on the dietary aspects. Contrary to expectations, no relationship was found between knowledge scores and economic level, literacy or formal education. This contrasts with studies in the USA ${ }^{6}, \mathrm{UK}^{8}$ and South Africa ${ }^{6}$ that reported a relationship between formal education and knowledge. However, women who worked outside the home had better knowledge for two scores, suggesting that contact with the wider community is perhaps more important in determining 
Table 5 Scores for the six domains of knowledge measured

\begin{tabular}{lccccc}
\hline Knowledge domain score & $n$ & Mean score* $^{*}$ & 25th percentile & Median† & 75th percentile \\
\hline Score 1: Diet- and behaviour-related causes of NCD & 299 & 47.0 & 33.3 & 44.4 & 61.1 \\
Score 2: Diet quality-NCD relationship & 299 & 37.8 & 15.4 & 38.5 & 53.9 \\
Score 3: Fruit and vegetable-NCD relationship & 300 & 19.9 & 0 & 0 & 33.3 \\
Score 4: Health consequences of obesity & 301 & 65.4 & 50.0 & 66.7 & 83.3 \\
Score 5: Causes of CVD & 299 & 60.6 & 45.5 & 63.6 & 81.8 \\
Score 6: Causes of certain cancers & 300 & 36.1 & 16.7 & 33.3 & 50.0 \\
\hline
\end{tabular}

NCD - non-communicable disease; CVD - cardiovascular disease.

* Mean percentage of correct responses.

$\dagger$ Median percentage of correct responses.

current knowledge than previous formal education or literacy level. It was also anticipated that obese women would have a better knowledge of the causes and consequences of obesity, but this was not the case; one possible explanation could be that as over $45 \%$ of women are overweight or obese, the condition is so widespread that all women have direct experience of overweight/obesity.

The questionnaire successfully measures six domains of knowledge of the diet and lifestyle-NCD relationship, as they passed criteria for content validity, item discrimination, item difficulty and internal consistency. This knowledge questionnaire could also be used in other countries, including those where low literacy is prevalent, as items are not only specific to Senegal. However, it would need to be tested for item discrimination and content validity in the target population. There are limitations of the tool. Due to the small sample of women used for piloting and time constraints in conducting fieldwork, construct validity tests were conducted after data collection on the whole sample of women in the final study, rather than on the pilot sample. Even so, the fact that the tool passed these criteria in the whole sample does not reduce its content validation.

The findings suggest that education targeting the benefits of vegetables and fruit may have the greatest impact on knowledge, given the general low understanding. A recent review of fruit and vegetable consumption patterns in subSaharan Africa ${ }^{24}$ reported low consumption, and our study of fruit and vegetable intake in Senegalese women concurs with this (data not presented here). A further reason for focusing on awareness of the benefits of fruit and vegetables is that increasing intake could have a potentially high impact in reducing many NCDs ${ }^{25}$. However, nutrition education messages need to be communicated within the context of a whole diet approach ${ }^{9}$ supported by simple, clear, positive food-based messages ${ }^{26}$ and take account of attitudes, risk perception and social norms, e.g. the desirability of overweight body sizes in urban women is a potential barrier to change in Senegal ${ }^{16}$. It may also be important to target those areas of knowledge which are well understood by the population as these may be more malleable to behaviour change. Women's knowledge was of particular relevance given their role in food purchasing and cooking responsibilities. However, future studies need to evaluate men's knowledge to identify if the pattern is similar, to inform a population-wide approach to improving knowledge.

This will require the development, evaluation and promotion of food-based dietary guidelines, culturally relevant to Senegal, as a tool for nutrition education; using a similar development process to that of other countries $^{27-29}$. Practical education strategies such as cooking, tasting and eating may be most effective, as education without associated skills development is likely to result in limited behaviour change.

\section{Acknowledgements}

The authors are grateful to the women of Pikine for participating so willingly in this study and to both Mame Sylla Diagne and Khady Diop for their invaluable work in the field.

\section{References}

1 World Health Organization (WHO). Global Strategy on Diet, Physical Activity and Health. Geneva: WHO, 2004.

2 Caballero B, Popkin BM. The Nutrition Transition: Diet and Disease in the Developing World. New York: Academic Press, 2002.

3 Delpeuch F, Maire B. Obesity and developing countries of the south. Médecine Tropicale 1997; 57: 380-8.

4 World Health Organization (WHO). Global Strategy on Diet, Physical Activity and Health: Africa Regional Consultation Meeting Report. Harare: WHO, 2003.

5 Nyamwaya D. Health promotion in Africa: strategies, players, challenges and prospects. Health Promotion International 2003; 18: 85-7.

6 Peltzer K. Nutrition knowledge among a sample of urban black South Africans. South African Journal of Clinical Nutrition 2004; 17: 24-31.

7 Holdsworth M, Haslam C, Raymond NT. Does the Heartbeat Award Scheme change employees' dietary attitudes and knowledge? Appetite 2000; 35: 179-88.

8 Parmenter K, Waller J, Wardle J. Demographic variation in nutrition knowledge in England. Health Education Research 2000; 15: 163-74.

9 Food and Agriculture Organization of the United Nations (FAO)/World Health Organization (WHO). Fruit and Vegetables for Health: Report of a Joint FAO/WHO Workshop. Geneva: WHO, 2004

10 Green LW, Kreuter MW. Health Promotion Planning: an Educational and Ecological Approach. Mountain View, CA: Mayfield Publishing, 1999.

11 McCaffery K, Wardle J, Waller J. Knowledge, attitudes and behavioural intentions in relation to early detection of 
colorectal cancer in the United Kingdom. Preventive Medicine 2003; 36: 525-35.

12 Anderson AS, Cox DN, McKellar S, Reynolds J, Lean ME, Mela DJ. Take 5, a nutrition education intervention to increase fruit and vegetable intakes: impact on attitudes towards dietary change. British Journal of Nutrition 1998; 80: $133-40$

13 Parmenter K, Wardle J. Development of a general nutrition knowledge questionnaire for adults. European Journal of Clinical Nutrition 2000; 53: 298-308.

14 Anderson AS, Bell A, Adamson A, Moynihan P. A questionnaire assessment of nutrition knowledge validity and reliability issues. Public Health Nutrition 2002; 5: $497-503$.

15 Whati LH, Seneka M, Steyn NP, Nel JH, Lombard C, Norris S. Development of a reliable and valid nutritional knowledge questionnaire for urban South African adolescents. Nutrition 2005; 24: 76-85.

16 Holdsworth M, Gartner A, Landais E, Maire B, Delpeuch F. Perceptions of healthy and desirable body size in urban Senegalese women. International Journal of Obesity and Related Metabolic Disorders 2004; 28: 1561-8.

17 Swift JA, Glazebrook C, Macdonald I. Development of a short scale for adults to assess knowledge regarding the health risks of obesity. International Journal of Obesity and Related Metabolic Disorders 2003; 27: S94.

18 Streiner DL, Norman GR. Health Measurement Scales: A Practical Guide to Their Development and Use. New York: Oxford University Press, 1995.

19 Kelley TL. The selection of upper and lower groups for the validation of test items. Journal of Educational Psychology 1939; 30: $17-24$

20 Kline P. The Handbook of Psychological Testing. London: Routledge, 1993.
21 Martin-Prevel YP, Traissac P, Delpeuch F, Maire B. Decreased attendance at routine health activities mediates deterioration in nutritional status of young African children under worsening socioeconomic conditions. International Journal of Epidemiology 2001; 30: 493-500.

22 Thioulouse J, Chessel D, Dolédec S, Olivier JM. ADE-4: a multivariate analysis and graphical display software. Statistics and Computing 1997; 7: 75-83.

23 Sapp S, Jensen H. Reliability and validity of nutrition knowledge and diet-health awareness test developed from the 1989-1991 diet and health survey. Journal of Nutrition Education 1997; 29: 63-72.

24 Ruel MT, Minot N, Smith L. Patterns and Determinants of Fruit and Vegetable Consumption in sub-Sabaran Africa: A Multicountry Comparison. Geneva: World Health Organization, 2005

25 Lock K, Pomerleau J, Causer L, Altmann DR, McKee M. The global burden of disease attributable to low consumption of fruit and vegetables: implications for the global strategy on diet. Bulletin of the World Health Organization 2005; 83: $100-8$.

26 Kelly CNM, Stanner SA. Diet and cardiovascular disease in the UK: are the messages getting across? Proceedings of the Nutrition Society 2003; 62: 583-9.

27 Hunt P, Rayner M, Gatenby S. A national food guide for the UK? Background and development. Journal of Human Nutrition and Dietetics 1995; 8: 315-22.

28 World Health Organization (WHO). Preparation and Use of Food Based Dietary Guidelines. Report of a Joint FAO/WHO Consultation, Nicosia, Cyprus. Geneva: WHO, 1996.

29 Vorster HH, Love P, Browne C. Development of food-based dietary guidelines for South Africa - the process. South African Journal of Clinical Nutrition 2001; 14: S3-6. 